\title{
Crenças e atitudes de educadores da rede pública de um município mineiro sobre o uso de Substâncias Psicoativas
}

\author{
Attitudes and beliefs of the public network educators about the use of \\ Psychoactive Substances
}

Marcus Luciano de Oliveira Tavares ${ }^{1,2}$, Amanda Márcia dos Santos Reinaldo², Belisa Vieira da Silveira ${ }^{3}$, Maria Odete Pereira²

${ }^{1}$ Faculdade Pitágoras de Belo Horizonte (FPBH) - Belo Horizonte (MG), Brasil

Universidade Federal de Minas Gerais (UFMG) - Belo Horizonte (MG), Brasil

${ }^{3}$ Universidade de São Paulo (USP) - São Paulo (SP), Brasil

\section{RESUMO}

Introdução: O despreparo de educadores para trabalhar com estudantes em uso de Substâncias Psicoativas é uma fragilidade que compromete a abordagem em ambiente escolar. Assim, é necessário que esses profissionais reconheçam a importância do conhecimento e reflitam suas crenças e atitudes acerca dessa temática. Objetivo: Conhecer as crenças e atitudes de educadores de nove escolas municipais de Belo Horizonte sobre o uso de Substâncias Psicoativas. Método: Estudo transversal, descritivo e exploratório realizado com 38 educadores da rede municipal de educação de Belo Horizonte/MG. Foi utilizado um questionário para caracterização dos dados sociodemográficos, a Escala de Tolerância Social e o Questionário sobre Modelo de Percepção de Problemas de Saúde. Resultados: Houve predominância de crenças negativas e estigmatizantes e atribuição do uso de Substâncias Psicoativas ao modelo moralizante. Conclusão: Intervenções focadas na conscientização de educadores sobre o uso e abuso de SPAs previamente à elaboração de programas de enfrentamento para escolares são necessárias para evitar situações conflitantes que estigmatizem o usuário.

Palavras-chave: drogas ilícitas; bebidas alcoólicas; tabaco; estigma social; professores escolares; transtornos relacionados ao uso de substâncias.

DOI: https://dx.doi.org/10.7322/abcshs.v44i1.1166

\section{ABSTRACT}

Introduction: The lack of preparation of educators to work with students using Psychoactive Substances is a fragility that compromises the approach in the school environment. Therefore, it is necessary that these professionals recognize the importance of knowledge and reflect their beliefs and attitudes about this subject. Objective: To know the beliefs and attitudes of educators of nine municipal schools in Belo Horizonte about the use of Psychoactive Substances. Methods: Cross-sectional, descriptive and exploratory study carried out with 38 educators from the municipal education network of Belo Horizonte/MG. The instruments used were a questionnaire to characterize sociodemographic data, the Social Tolerance Scale and the Questionnaire on Perception of Health Problems Questionnaire. Results: There was a predominance of negative and stigmatizing beliefs and attribution of the use of Psychoactive Substances to the moralizing model. Conclusion: Interventions focused on educators' awareness about the use and abuse of SPAs prior to the elaboration of coping programs for schoolchildren are necessary to avoid conflicting situations that stigmatize the user.

Keywords: street drugs; alcoholic beverages; tobacco; social stigma; school teachers; substance-related disorders. 


\section{INTRODUÇÃO}

No Brasil, o início do uso de Substâncias Psicoativas (SPAs) ocorre durante a adolescência, sendo o álcool aos 13 e as demais drogas entre os 13 e 14 anos. Uma das principais causas evitáveis de morte entre a população de 15 a 24 anos está relacionada ao uso e abuso de SPAs ${ }^{1}$.

A prevenção ao uso de SPAs em escolas é uma estratégia utilizada em todo o mundo com vistas a promover a saúde entre crianças e adolescentes. Por ser um dos locais onde esses indivíduos permanecem vinculados por tempo considerável e convivem em sociedade, a escola é considerada local estratégico ${ }^{2}$.

Frequentemente, as escolas são pressionadas a desenvolver seus próprios programas de prevenção ao uso de SPAs devido à demanda que seus alunos apresentam, dessa maneira, esses são expostos a diferentes programas ao longo de sua vida escolar, dificultando o vínculo ou um processo de avaliação contínuo dessas intervenções ${ }^{3}$.

Outra fragilidade existente na abordagem do uso de SPAs em escolas é a falta de preparo dos educadores sobre essa temática. Esses profissionais apresentam despreparo para abordagem do assunto, bem como crenças estigmatizantes, o que influencia a existência de atitudes negativas, comprometendo a maneira de trabalhar a temática com os alunos ${ }^{4,5}$.

Baseado nisso, consideramos que a elaboração de um programa de intervenção eficaz tem início no conhecimento prévio sobre as fragilidades e potencialidades dos indivíduos envolvidos nesse processo, portanto, é necessário conhecer as crenças e atitudes dos educadores em relação ao uso de SPAs, pois dessa maneira pode-se intervir nas fragilidades existentes. Dado o exposto, tomamos por objetivo conhecer as crenças e atitudes de educadores de nove escolas municipais de Belo Horizonte sobre o uso de SPAs.

\section{MÉTODO}

Trata-se de um estudo transversal, descritivo, realizado no município de Belo Horizonte, Minas Gerais, Brasil.

O município é subdividido em nove unidades administrativas, sendo elas: Barreiro, Centro Sul, Leste, Nordeste, Noroeste, Norte, Oeste, Pampulha e Venda-Nova. O estudo foi realizado com educadores participantes das oficinas sobre Álcool e outras Drogas.

Os educadores participantes são lotados em nove escolas públicas vinculadas à Secretaria Municipal de Educação (SME) de Belo Horizonte. A seleção das escolas deu-se por conveniência. Foi escolhida uma escola de cada unidade administrativa do município.

O processo para seleção dos educadores obedeceu aos seguintes critérios: funcionários ativos e efetivos, que faltassem maior tempo para aposentadoria e tivessem menor percentual de absenteísmo, pois dessa forma, favoreceria a etapa de implementação do projeto a ser desenvolvido. Optamos por selecionar três funcionários de cada escola, sendo um professor, um gestor e um monitor do Programa Saúde na Escola (PSE). Participaram, ainda, 11 educadores vinculados ao Grupo de Trabalho (GT) sobre o uso abusivo de Álcool e outras Drogas para a educação da SMEBH. Esse GT é composto por gestores da área da educação e foi criado com o intuito de trabalhar sobre a temática envolvendo o uso de SPAs por meio do desenvolvimento de estratégias a serem implementada na rede. Por fim, a amostra foi constituída por 38 educadores, os quais foram convocados pela SME para participação nas oficinas.

A coleta de dados ocorreu em agosto de 2016, na data de realização da primeira oficina de capacitação sobre uso de SPAs, nesse momento, foram apresentados os objetivos do estudo aos envolvidos, sendo feito o convite para participação na pesquisa. Utilizamos como instrumento três questionários autoaplicáveis que foram respondidos previamente a realização da capacitação para essa não se configurasse como um viés, induzindo as respostas dos educadores. Os questionários aplicados foram:

Questionário Sóciodemográfico: elaborados pela equipe de pesquisa, foi composto por questões referentes ao sexo, idade, estado civil, tempo de profissão, se teve contato com usuário em uso problemático de drogas no último ano e uma pergunta sobre o nível de motivação do educador em participar do projeto de intervenção;

Escala de Tolerância Social: elaborado por Silveira et al. ${ }^{6}$, possui objetivo de avaliar a aprovação ou reprovação dos sujeitos quanto ao uso de drogas e comportamentos de risco por meio de respostas do tipo Likert, variando de um (desaprovo totalmente) a cinco (aprovo totalmente). Foram calculadas as médias dos resultados, as quais possibilitaram apontar comportamentos aceitos ou reprovados pelos educadores.

Questionário sobre modelo de percepção de problemas de saúde: esse questionário investiga a opinião dos sujeitos quanto à responsabilidade de um indivíduo pelo acometimento e solução de determinadas condições de saúde, as respostas às questões eram em formato Likert, variando de um (nada responsável) a cinco (totalmente responsável). Tais questões serviram de base para a construção dos modelos propostos por Brickman, em que as respostas são classificadas em quatro modelos: Modelo Médico: a causa da condição foge da alçada do indivíduo, sendo este pouco ou nada responsável, a condição seria de origem biológica, dessa forma, a responsabilidade pela solução ou controle não seria do indivíduo; Modelo Moralista: propõe que a responsabilidade pelo aparecimento e solução de uma condição é atribuída ao indivíduo; Modelo Compensatório: o aparecimento do problema não é de responsabilidade do indivíduo, porém sua solução é atribuída a ele; Modelo Iluminado: neste modelo, a responsabilidade pelo aparecimento do problema é do indivíduo, entretanto, a solução não depende do mesmo ${ }^{6,7}$.

Para elaboração do banco de dados e análise dos dados utilizamos os programas estatísticos EpiInfo ${ }^{\mathrm{Tx}} 7.2$ e o Data 
Analysis Statistical Software (STATA) versão 14.0. A análise foi realizada por meio de frequência absoluta e relativa para as variáveis categóricas.

O projeto foi aprovado pelo Comitê de Ética em Pesquisa da Universidade Federal de Minas Gerais sob Parecer $\mathrm{n}^{\circ}$ 37574914.3.0000.5149, e seguiu os aspectos éticos baseados na Resolução no 466/12 do Conselho Nacional de Saúde, incluindo a apresentação do Termo de Consentimento Livre Esclarecido. Todos os participantes tiveram sigilo garantido e resguardado em todas as etapas da pesquisa.

\section{RESULTADOS}

Na Tabela 1, encontram-se as variáveis de caracterização sociodemográfica. Percebe-se predominância do sexo feminino, profissionais na faixa etária de 40 a 59 anos, casados ou que vivem com companheiro, com 15 a 30 anos de profissão, que conviveram com algum usuário de drogas nos últimos 12 meses e que se consideram motivados para trabalhar com alunos em uso prejudicial de SPAs.

Na Tabela 2 estão elencadas as frequências referentes à Escala de Tolerância Social, destacando-se o elevado percentual de

Tabela 1: Caracterização sociodemográfica dos educadores, Belo Horizonte, $2016(n=38)$

\begin{tabular}{|c|c|c|}
\hline Variáveis & $\mathbf{n}$ & $\%$ \\
\hline \multicolumn{3}{|l|}{ Sexo } \\
\hline Feminino & 30 & 79,0 \\
\hline Masculino & 8 & 21,0 \\
\hline \multicolumn{3}{|l|}{ Idade } \\
\hline 18 a 29 & 5 & 13,2 \\
\hline 30 a 39 & 8 & 21,0 \\
\hline 40 a 59 & 24 & 63,2 \\
\hline$\geq 60$ & 1 & 2,6 \\
\hline \multicolumn{3}{|l|}{ Estado Civil } \\
\hline Solteiro & 12 & 31,6 \\
\hline Casado/Vive com companheiro & 23 & 60,5 \\
\hline Divorciado & 3 & 7,9 \\
\hline Viúvo & - & - \\
\hline \multicolumn{3}{|l|}{ Tempo de profissão } \\
\hline 1 a 5 anos & 9 & 23,7 \\
\hline 6 a 15 anos & 8 & 21 \\
\hline 15 a 30 anos & 17 & 44,8 \\
\hline$\geq 31$ anos & 4 & 10,5 \\
\hline \multicolumn{3}{|l|}{$\begin{array}{l}\text { Convívio com usuário de SPAs no } \\
\text { último ano }\end{array}$} \\
\hline Não & 7 & 18,4 \\
\hline Sim & 31 & 81,6 \\
\hline \multicolumn{3}{|l|}{$\begin{array}{l}\text { Motivação para trabalhar com alunos } \\
\text { em uso prejudicial de SPAs }\end{array}$} \\
\hline Totalmente desmotivado & 3 & 7,9 \\
\hline Desmotivado & 3 & 7,9 \\
\hline Neutro & 6 & 15,8 \\
\hline Motivado & 19 & 50,0 \\
\hline Totalmente motivado & 7 & 18,4 \\
\hline
\end{tabular}

educadores indiferentes quanto ao sentimento de indiferença sobre consumir bebidas alcoólicas (39,5\%).

Na Figura 1, pode-se visualizar o valor médio dos sentimentos dos educadores em relação à aprovação/reprovação do uso de SPAs e comportamentos de risco para o desenvolvimento de doenças e outros, a escala varia de um (reprovação total) a cinco (aprovação total).

$\mathrm{Na}$ Tabela 3, estão expostos os dados individuais referentes às dimensões propostas pelo Modelo de Percepção de Brickman. Os valores totais de " $n$ " para cada condição podem diferir, uma vez que a opção "neutra" foi eliminada da análise, portanto a interpretação deve ser feita por meio do valor percentual.

\section{DISCUSSÃO}

A abordagem da temática relacionada ao uso e abuso de SPAs deve ser realizada continuamente com educadores, pois são profissionais que possuem contato frequente com adolescentes, indivíduos que comumente sentem-se pressionados a assumir comportamentos e tomar decisões impostas pelo seu meio social, o que pode resultar em comportamentos de risco. Empoderar os educadores do conhecimento necessário para lidar com a

Tabela 2: Frequência de aprovação/reprovação em relação a hábitos e comportamentos de outras pessoas, Belo Horizonte, $2016(n=38)$

\begin{tabular}{|l|c|c|c|c|c|c|}
\hline \multirow{2}{*}{ Hábitos/comportamentos } & \multicolumn{2}{|c|}{ Reprova } & \multicolumn{2}{|c|}{ Indiferente } & \multicolumn{2}{|c|}{ Aprova } \\
\hline & $\mathbf{n}$ & $\%$ & $\mathbf{n}$ & $\%$ & $\mathbf{n}$ & $\%$ \\
\hline Fumar & 31 & 81,6 & 5 & 13,2 & 2 & 5,3 \\
\hline Consumir bebidas alcoólicas & 17 & 44,7 & 15 & 39,5 & 6 & 15,8 \\
\hline Jogos de azar & 27 & 71 & 8 & 21,1 & 3 & 7,9 \\
\hline Fumar maconha & 30 & 79 & 7 & 18,4 & 1 & 2,6 \\
\hline Usar cocaína & 34 & 89,4 & 2 & 5,3 & 2 & 5,3 \\
\hline Beber e dirigir & 34 & 89,4 & 2 & 5,3 & 2 & 5,3 \\
\hline $\begin{array}{l}\text { Atividades sexuais } \\
\text { sem preservativo }\end{array}$ & 28 & 73,7 & 7 & 18,4 & 3 & 7,9 \\
\hline
\end{tabular}

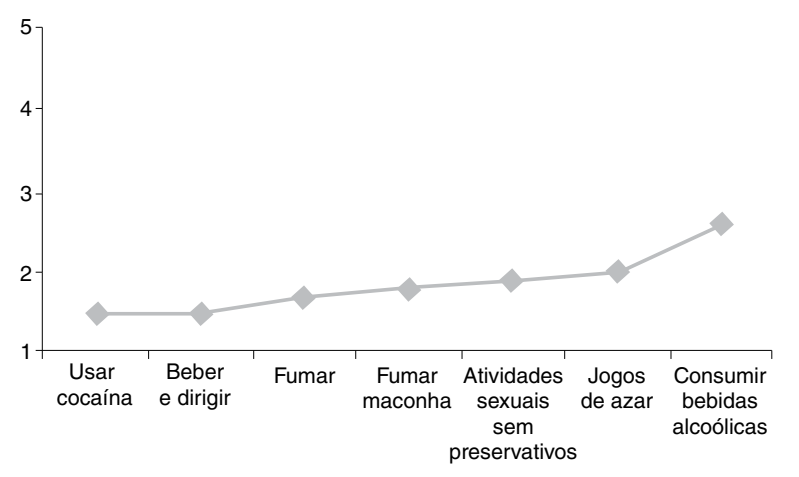

Figura 1: Média dos sentimentos de aprovação/reprovação em relação a hábitos e comportamentos de outras pessoas, Belo Horizonte, 2016. 
problemática relacionada ao uso de SPAs se traduzirá na redução de estigmas envolvendo a dependência. $\mathrm{O}$ estigma e o preconceito são barreiras para a abordagem do uso, pois se baseiam nas crenças e, por consequência, influenciam nas atitudes, o que repercute negativamente na abordagem do tema e, como consequência, dificulta a realização de intervenções aos dependentes químicos ${ }^{8}$.

A importância da capacitação de educadores sobre a temática já é percebida pela Tabela 1, em que $81,6 \%$ referiram convívio com usuários de SPAs nos últimos doze meses. Esse convívio pode se traduzir em momentos oportunos para intervenções passíveis de serem realizadas pelos educadores, porém a capacitação prévia é imprescindível, visto que as crenças e atitudes desses profissionais tendem a ser estigmatizantes ${ }^{4,9}$.

Percebe-se ainda que 50\% dos educadores se consideram motivados para o trabalho com alunos em uso prejudicial de SPAs, porém, na prática, ações de prevenção ao uso de drogas não são desenvolvidas, ou quando são, ocorrem de maneira descontinuada, com múltiplas abordagens ${ }^{9}$. Contudo, esse resultado deve ser visto como positivo, pois ao somar os que estão "motivados" e "totalmente motivados", observamos $68,4 \%$ de educadores dispostos a abordar essa temática que, muitas vezes, devido à sua intersetorialidade, se veem despreparados e impotentes ante o problema ${ }^{5}$.

O uso e abuso de álcool é apontado pela literatura como um dos principais fatores de risco para o desenvolvimento de inúmeras doenças e agravos, como acidentes de trânsito, violência e condições incapacitantes ${ }^{10,11}$. Pela Tabela 2, percebe-se que o consumo de bebidas alcoólicas obteve menor frequência de reprovação $(44,7 \%)$ em relação às demais SPAs, apresentando, ainda maiores valores de indiferença e aprovação. Tais resultados são explicados, possivelmente, por ser uma droga socialmente aceita e de fácil acesso. Pode-se inferir, ainda, que a elevada frequência de educadores indiferentes ao consumo está relacionada a dificuldade em se posicionar contra ou a favor, uma vez que se trata de uma droga menos estigmatizada.

Percebe-se um elevado percentual de reprovação em relação ao hábito de "beber e dirigir" (89,4\%), resultado visto como positivo,

Tabela 3: Valores individuais do Modelo de Percepção de Brickman, Belo Horizonte, 2016

\begin{tabular}{|l|c|c|c|c|c|c|c|c|}
\hline & \multicolumn{9}{|c|}{ Modelo de Percepção de Brickman } \\
\hline \multirow{2}{*}{ Condição } & Moral & Médico & lluminado & Compensatório \\
\hline & $\mathbf{n}$ & $\%$ & $\mathbf{n}$ & $\%$ & $\mathbf{n}$ & $\%$ & $\mathbf{n}$ & $\%$ \\
\hline Diabetes & 6 & 37,5 & 1 & 6,25 & - & & 9 & 56,25 \\
\hline Tabagismo & 19 & 79,1 & 1 & 4,2 & 1 & 4,2 & 3 & 12,5 \\
\hline Esquizofrenia & - & - & 17 & 77,3 & 3 & 13,6 & 2 & 9,1 \\
\hline Hanseníase & 2 & 11,1 & 11 & 61,1 & - & - & 5 & 27,8 \\
\hline Alcoolismo & 16 & 80 & 1 & 5 & - & - & 3 & 15 \\
\hline Obesidade & 10 & 62,5 & - & - & - & - & 6 & 37,5 \\
\hline Depressão & 3 & 21,4 & 7 & 50 & - & & 4 & 28,6 \\
\hline AlDS & 8 & 38,1 & 1 & 4,8 & 10 & 47,6 & 2 & 9,5 \\
\hline $\begin{array}{l}\text { Dependência } \\
\text { da maconha/ }\end{array}$ & 13 & 68,5 & 2 & 10,5 & 2 & 10,5 & 2 & 10,5 \\
\hline cocaína & & & & & & & & \\
\hline
\end{tabular}

pois tal hábito está associado como uma das principais causas de acidentes de trânsito no mundo ${ }^{12}$, porém nos leva a refletir sobre uma incompatibilidade com a frequência apresentada para a reprovação do consumo de bebidas alcoólicas. A reprovação para o hábito de beber e dirigir é de aproximadamente o dobro da reprovação para o consumo de bebidas alcoólicas, portanto, esperava-se valores aproximados de reprovação entre as duas variáveis, uma vez que ambas podem coexistir, o que não aconteceu.

O hábito de fumar foi reprovado pela maioria dos educadores, tal resultado pode ser compreendido como um reflexo das políticas e programas de regulação e proibição do seu uso, fatores que levaram a população a repensar sobre os riscos associados ao uso dessa substância

Destacam-se os elevados percentuais de reprovação para o uso de cocaína $(89,4 \%)$ e da maconha (79\%). Usuários dessas drogas são frequentemente associados à marginalização, comportamentos violentos e mais estigmatizados que os usuários de álcool, este que teve menor reprovação $(44,7 \%)$ e maior aprovação $(15,8 \%)$ que os demais hábitos e comportamentos, tais resultados, possivelmente, estão relacionados ao estigma recorrente sobre os usuários dessas substâncias ${ }^{13}$.

Ainda que não seja o enfoque deste estudo, preocupou-nos perceber que a prática de atividades sexuais sem preservativo foi aprovada por $7,9 \%$ e tratada com indiferença por $18,4 \%$ dos educadores. Esse resultado revela a necessidade de abordar essa temática com educadores, pois trata-se de outro tema frequentemente negligenciado em escolas, passível de repercussões sociais e de saúde.

A Tabela 3 refere-se ao Modelo Geral de Percepção de Brickman e mostra o quanto os educadores atribuem o alcoolismo (80\%), tabagismo $(79,1)$ e dependência da maconha/cocaína ao modelo moral $(68,5 \%)$ ao modelo moral.

Esses resultados traduzem a visão negativa que a dependência química possui, uma vez que, dentre as condições de saúde analisadas, apresentam maiores percentuais compreendidos no modelo moral. A causa desses comportamentos está frequentemente associada à fraqueza moral e, sua resolubilidade unicamente à força de vontade, isso pode influenciar nas atitudes desses profissionais, pois se tratam de crenças estigmatizantes da dependência química. $\mathrm{O}$ fato de a dependência de SPAs seguir um modelo moralizado pode impactar negativamente na maneira com que os educadores lidam com a problemática ${ }^{9-14}$.

Araldi et $a l .{ }^{4}$, em seu estudo sobre as representações sociais de educadores a respeito do uso de SPAs apontaram uma visão estigmatizante da adolescência e do uso de álcool e outras drogas nessa faixa etária, concluindo que isso "dificulta o diálogo aberto sobre essa questão com os adolescentes e uma atuação de prevenção nas escolas".

Intervenções focadas na redução dos estigmas acerca do uso de SPAs são necessárias para controle das repercussões negativas 
causadas na vida dos usuários, pois o estigma está associado à redução da autoestima, aumento da depressão e ansiedade. O apoio social aos usuários de SPAs é um fator positivo para o enfrentamento dos impactos negativos do estigma e da vergonha internalizada na saúde mental e no bem-estar social ${ }^{15,16}$.

Assim conclui-se que esse estudo, que teve como objetivo conhecer as crenças e atitudes de educadores de nove escolas municipais de Belo Horizonte sobre o uso de SPAs, revelou a maioria dos educadores encontram-se motivados para o trabalho com alunos em uso prejudicial de SPAs, porém, ao mesmo tempo, vinculam o uso de SPAs ao modelo moralizante, além de apresentar maiores frequências de aprovação para o uso de álcool, enquanto maiores reprovações para o uso de tabaco, maconha e cocaína, todas consideradas nocivas.

Intervenções focadas na conscientização de educadores sobre o uso e abuso de SPAs previamente à elaboração de programas de prevenção para escolares são necessárias para evitar situações conflitantes que estigmatizem o usuário.

\section{REFERÊNCIAS}

1. Carlini ELA, Noto AR, Sanchez ZM, Carlini CMA, Locatelli DP, Abeid LR, et al. VI Levantamento Nacional sobre o Consumo de Drogas Psicotrópicas entre estudantes do ensino fundamental e médio das redes pública e privada de ensino nas 27 capitais brasileiras: 2010. Brasília: SENAD; 2010.

2. Onrust SA, Otten R, Lammers J, Smit F. School-based programmes to reduce and prevent substance use in different age groups: What works for whom? Systematic review and meta-regression analysis. Clin Psychol Rev. 2016;44:45-59. http://dx.doi.org/10.1016/j.cpr.2015.11.002

3. Kumar R, O'Malley P, Lloyd J, Viginia L. Alcohol, Tobacco, and Other Drug Use Prevention Programs in U.S. Schools: A Descriptive Summary. Prev Sci. 2013;14(6):581-92. http://dx.doi.org/10.1007/s11121-012-0340-z

4. Araldi JC, Njaine K, Oliveira MC, Ghizoni AC. Representações sociais de professores sobre o uso abusivo de álcool e outras drogas na adolescência: repercussões nas ações de prevenção na escola. Interface. 2012;16(40):135-48.

http://dx.doi.org/10.1590/S1414-32832012005000002

5. Knevitz MF, Béria JU, Schermann LB. Percepções e demandas de professores sobre educação preventiva ao abuso de álcool e outras drogas. Holos. 2017;4:357-70.

https://doi.org/10.15628/holos.2017.4901

6. Silveira OS, Ronzani TM, Bastos RR. Estigmatização do uso de álcool e outras drogas entre profissionais de saúde de Juiz de Fora. Dissertação (Mestrado) - Universidade Federal de Juiz de Fora. Juiz de Fora: 2010.

7. Palm J. Moral concerns: treatment staff and user perspectives on alcohol and drug problems. Tese (Doutorado) - University of Stockholm. Stockholm: 2006.

8. Livingston JD, Milne T, Fang ML, Amari E. The effectiveness of interventions for reducing stigma related to substance use disorders: a systematic review. Addiction. 2012 107(1):39-50

http://dx.doi.org/10.1111/j.1360-0443.2011.03601.x

9. Moreira A, Vóvio CL, Micheli D. Prevenção ao consumo abusivo de drogas na escola: desafios e possibilidades para a atuação do educador. Educ Pesqui. 2015;41(1):119-35 http://dx.doi.org/10.1590/S1517-97022015011670

10. Antunes F, Oliveira MLF. Characteristics of patients hospitalized at an intensive care unit due to drug abuse. Invest Educ Enferm. 2013;31(2):201-9

11. Lachenmeier DW, Rehm J. Comparative risk assessment of alcohol, tobacco, cannabis and other illicit drugs using the margin of exposure approach. Sci Rep. 2015;5:8126. http://dx.doi.org/10.1038/srep08126

12. Brady JE, Li G. Trends in Alcohol and Other Drugs Detected in Fatally Injured Drivers in the United States, 1999-2010. Am J Epidemiol. 2014;179(6):692-9. http://dx.doi.org/10.1093/aje/kwt327

13. Bard ND, Antunes B, Roos CM, Olschowsky A, Pinho LB. Stigma and prejudice: the experience of crack users. Rev Latino-Am Enfermagem. 2016;24:e2680.

http://dx.doi.org/10.1590/1518-8345.0852.2680

14. Barros JPP, Colaco VFR. Drogas na Escola: análise das vozes sociais em jogo. Educ Real. 2015;40(1):253-73. http://dx.doi.org/10.1590/2175-623644605

15. Birtel MD, Wood L, Kempa NJ. Stigma and social support in substance abuse: Implications for mental health and well-being. Psychiatry Res. 2017;252:1-8.

http://dx.doi.org/10.1016/j.psychres.2017.01.097

16. Frank LE, Nagel SK. Addiction and moralization: the role of the underlying model of addiction. Neuroethics. 2017;10(1):129-39. http://dx.doi.org/10.1007/s12152-017-9314-y 